\title{
KOLMOGOROV-SMIRNOV ISOMETRIES OF THE SPACE OF GENERALIZED DISTRIBUTION FUNCTIONS
}

\author{
LAJOS MOLNÁR* AND PATRĆIA SZOKOL**
}

\begin{abstract}
In this paper we describe the structure of surjective isometries of the space of all generalized probability distribution functions on $\mathbb{R}$ with respect to the Kolmogorov-Smirnov metric.
\end{abstract}

The study of linear isometries of linear function spaces and operator spaces has been an extensive research area in functional analysis over the past decades. The starting point of those investigations is the famous Banach-Stone theorem which describes the structure of all surjective linear isometries between the Banach spaces of complex-valued continuous functions on compact Hausdorff spaces equipped with the supremum norm. One can find a comprehensive and very nice overview of the topic in the two volume monograph [3], [4].

However, there are important metric spaces of functions which are not linear spaces. For example, the set of all probability distribution functions on $\mathbb{R}$ that plays so fundamental role in probability theory and statistics is not a linear space. By a distribution function here we mean a mapping $d: \mathbb{R} \rightarrow \mathbb{R}$ which is monotone increasing, continuous from the right, and has limit 0 at $-\infty$ and 1 at $\infty$. The set of all such functions is denoted by $D(\mathbb{R})$. Motivated by the famous Banach-Stone theorem, in the recent paper [1] G. Dolinar and the first author have described the general forms of surjective isometries of $D(\mathbb{R})$ with respect to the Kolmogorov-Smirnov metric. This metric originates from the uniform norm on the Banach space of all real-valued bounded functions of a real variable. Namely, for any $f, g \in D(\mathbb{R})$ the Kolmogorov-Smirnov distance $\rho(f, g)$ between $f$ and $g$ is defined by the formula

$$
\rho(f, g)=\sup _{t \in \mathbb{R}}|f(t)-g(t)| .
$$

2010 Mathematics Subject Classification. Primary: 47B38, 54E40.

Key words and phrases. Generalized probability distribution functions, KolmogorovSmirnov metric, isometry.

The first author was supported by the Hungarian Scientific Research Fund (OTKA), Grant No. K81166 NK81402, and also by the TÁMOP 4.2.1./B-09/1/KONV-2010-0007 project implemented through the New Hungary Development Plan co-financed by the European Social Fund and the European Regional Development Fund. 
The significance of this metric lies in its applications in the Kolmogorov-Smirnov statistic and test.

As mentioned above, the structure of all surjective isometries of the space of $D(\mathbb{R})$ has been described in [1]. We emphasize that no sort of linearity of the isometries has been assumed. The corresponding result reads as follows.

Theorem 1. ([1]) Let $\phi: D(\mathbb{R}) \rightarrow D(\mathbb{R})$ be a surjective isometry with respect to the Kolmogorov-Smirnov metric, i.e. assume that $\phi$ is a bijective map with the property that

$$
\rho(\phi(f), \phi(g))=\rho(f, g)
$$

holds for all $f, g \in D(\mathbb{R})$. Then either there exists a strictly increasing bijection $\varphi: \mathbb{R} \rightarrow \mathbb{R}$ such that $\phi$ is of the form

$$
\phi(f)(t)=f(\varphi(t)), \quad t \in \mathbb{R}, f \in D(\mathbb{R})
$$

or there exists a strictly decreasing bijection $\psi: \mathbb{R} \rightarrow \mathbb{R}$ such that $\phi$ is of the form

$$
\phi(f)(t)=1-f(\psi(t)-), \quad t \in \mathbb{R}, f \in D(\mathbb{R}) .
$$

Here $f(t-)$ denotes the left limit of the distribution function $f$ at the point $t \in \mathbb{R}$. Observe that similarly to the conclusion in Banach-Stone theorem these isometries are closely related to composition operators. The key step of the proof of the theorem above has been a metric characterization of Dirac distribution functions (i.e. distribution functions corresponding to one point mass measures).

The aim of the present work is to extend Theorem 1 to the larger space $\Delta(\mathbb{R})$ of all generalized probability distribution functions. This problem has been raised by our colleague Mátyás Barczy who works in probability theory. By a generalized distribution function we mean a function from $\mathbb{R}$ to $[0,1]$ which is monotone increasing and continuous from the right without restrictions on its limits at $\pm \infty$. Generalized distribution functions appear naturally when one considers random variables taking values not only in $\mathbb{R}$ but in the extended real line which setting proves useful in several investigations. In fact, some practical reasons are coming from measure theory (e.g., if one deals with extended realvalued functions, then in Beppo Levi theorem there is no need to assume the convergence of the series of non-negative measurable functions). Beside them we recall serious applications in the theory of renewal processes (see [2] where the word "defective" is used for random variables taking the value $\infty$ with positive probability and also for the relating distribution functions) or Helly's fundamental theorem on the weak sequential compactness of the space of generalized distribution functions (see, e.g., [6]) that plays an important role in probability theory. The Kolmogorov-Smirnov metric on $\Delta(\mathbb{R})$ is defined by the same formula as on $D(\mathbb{R})$.

Our result that follows describes the structure of surjective KolmogorovSmirnov isometries of the space of generalized distribution functions and shows 
that this structure is formally the same as that of the surjective isometries of $D(\mathbb{R})$. Namely, any surjective isometry of $\Delta(\mathbb{R})$ is induced either by a strictly increasing bijection or by a strictly decreasing bijection of $\mathbb{R}$.

Theorem 2. Let $\phi: \Delta(\mathbb{R}) \rightarrow \Delta(\mathbb{R})$ be a surjective isometry with respect to the Kolmogorov-Smirnov metric $\rho$. Then either there exists a strictly increasing bijection $\varphi: \mathbb{R} \rightarrow \mathbb{R}$ such that $\phi$ is of the form

$$
\phi(f)(t)=f(\varphi(t)), \quad t \in \mathbb{R}, f \in \Delta(\mathbb{R})
$$

or there exists a strictly decreasing bijection $\psi: \mathbb{R} \rightarrow \mathbb{R}$ such that $\phi$ is of the form

$$
\phi(f)(t)=1-f(\psi(t)-), \quad t \in \mathbb{R}, f \in \Delta(\mathbb{R}) .
$$

Before presenting the proof, observe that, as explained in [1], every transformation which appears on the right hand side of the two displayed formulas above is a surjective isometry of $\Delta(\mathbb{R})$. The content of our result is, in fact, that the reverse statement is also true: every surjective isometry of $\Delta(\mathbb{R})$ is necessarily of one of those two forms.

Proof. The basic idea of our proof is to find a way to deduce the statement from Theorem 1. All that follow are done for that purpose.

We start with presenting a metric characterization of the constant 0 and constant 1 functions. For any real number $c \in[0,1]$ let $\underline{c}$ denote the constant $c$ function. The closed ball with center $f \in \Delta(\mathbb{R})$ and radius $r \geq 0$ is denoted by $B_{r}(f)$. We now assert that for any $f, g \in \Delta(\mathbb{R})$ we have that $B_{1 / 2}(f) \cap B_{1 / 2}(g)$ is a singleton if and only if $\{f, g\}=\{\underline{0}, \underline{1}\}$. The sufficiency is obvious. To see the necessity, assume that $f, g \in \Delta(\mathbb{R})$ are such that $B_{1 / 2}(f) \cap B_{1 / 2}(g)$ is a singleton. We distinguish some cases.

(I) If

$$
|f(x)-g(x)|=1, \quad x \in \mathbb{R},
$$

then we easily get that $f=\underline{0}$ and $g=\underline{1}$, or that $f=\underline{1}$ and $g=\underline{0}$.

(II) Suppose that there exists an $x \in \mathbb{R}$ such that $|f(x)-g(x)|<1$ but for all such $x$ we have $f(x)=g(x)$. This means that for every real number $x$ either we have $|f(x)-g(x)|=1$ or we have $f(x)=g(x)$ and there do exist a real number $x_{0}$ such that the second condition holds. In what follows, by an identity point of $f$ and $g$ we mean a real number $x$ such that $f(x)=g(x)$ and by an extreme point of the function $h \in \Delta(\mathbb{R})$ we mean a point $x \in \mathbb{R}$ such that $h(x)=0$ or $h(x)=1$ holds. Now, if every identity point of $f$ and $g$ is an extreme point of $f$ and $g$, then in the present case we obtain that every point is an extreme point of both $f$ and $g$. This implies that $f$ (and also $g$ ) must be a Dirac distribution function, or $\underline{0}$, or $\underline{1}$. On the other hand, if there exists an identity point of $f$ and $g$ where the corresponding common value of the functions is in ]0,1[, then one can easily verify that $f=g$ which obviously contradicts the fact that $B_{1 / 2}(f) \cap B_{1 / 2}(g)$ 
is a singleton. We arrive at the same contradiction when both $f$ and $g$ are Dirac distribution functions, and also when one of them is a Dirac distribution function and the other one is either $\underline{0}$ or $\underline{1}$. It follows that $\{f, g\} \subset\{\underline{0}, \underline{1}\}$ and since $f \neq g$, the desired conclusion follows in the case (II).

(III) It remains to consider the case where there exists a real number $x_{0}$ such that

$$
\left|f\left(x_{0}\right)-g\left(x_{0}\right)\right|<1 \text { and } f\left(x_{0}\right) \neq g\left(x_{0}\right) .
$$

Without loss of generality we may and do suppose that $0<f\left(x_{0}\right)-g\left(x_{0}\right)<1$. Obviously,

$$
h(x) \doteq \frac{f(x)+g(x)}{2}, \quad x \in \mathbb{R}
$$

is an element of $B_{1 / 2}(f) \cap B_{1 / 2}(g)$. We are going to show that this function can be modified on a short interval such that the so obtained function also belongs to $B_{1 / 2}(f) \cap B_{1 / 2}(g)$. Since $f, g$ and $h$ are continuous from the right, there exists $\epsilon>0$ such that

$$
\begin{array}{rlrl}
h\left(x_{0}+\epsilon\right) & <f\left(x_{0}\right), & g\left(x_{0}+\epsilon\right) & <h\left(x_{0}\right), \\
f\left(x_{0}+\epsilon\right)-\frac{1}{2}<h\left(x_{0}\right), & & h\left(x_{0}+\epsilon\right)<g\left(x_{0}\right)+\frac{1}{2} .
\end{array}
$$

(a) Suppose that $h\left(x_{0}\right)<h\left(x_{0}+\epsilon\right)$. Let $h_{1}$ be any generalized distribution function with $h_{1}\left(x_{0}\right)=h\left(x_{0}\right)$ which may differ from the function $h$ only on the interval $\left[x_{0}, x_{0}+\epsilon[\right.$. From the inequalities above it follows that

$0<h\left(x_{0}\right)-g\left(x_{0}+\epsilon\right) \leq h_{1}(t)-g(t) \leq h\left(x_{0}+\epsilon\right)-g\left(x_{0}\right)<\frac{1}{2}, \quad t \in\left[x_{0}, x_{0}+\epsilon[\right.$

and

$0<f\left(x_{0}\right)-h\left(x_{0}+\epsilon\right) \leq f(t)-h_{1}(t) \leq f\left(x_{0}+\epsilon\right)-h\left(x_{0}\right)<\frac{1}{2}, \quad t \in\left[x_{0}, x_{0}+\epsilon[\right.$

which means that $h_{1} \in B_{1 / 2}(f) \cap B_{1 / 2}(g)$. Observe that there are infinitely many such functions $h_{1}$.

(b) In what follows we analyze the case where

$$
h\left(x_{0}\right)=h\left(x_{0}+\epsilon\right) .
$$

Consider the set of all real numbers $x$ such that $x_{0} \leq x$ and $h\left(x_{0}\right)=h(x)$ (we also have $f\left(x_{0}\right)=f(x)$ and $\left.g\left(x_{0}\right)=g(x)\right)$. These points form an interval. In the case where this interval is not bounded, we have that $f$ and $g$ are constant functions on $\left[x_{0}, \infty[\right.$. It follows easily that on this interval one can change the constant value of $h$ to other constants such that the so obtained functions are elements of $B_{1 / 2}(f) \cap B_{1 / 2}(g)$, a contradiction.

If the interval above is bounded, then denote by $x_{1}$ its supremum. If $h\left(x_{1}\right)>$ $h\left(x_{0}\right)$, then the constant value of the function $h$ on $\left[x_{0}, x_{1}\right.$ [ can be changed to obtain different elements of $B_{1 / 2}(f) \cap B_{1 / 2}(g)$, a contradiction. Assuming $h\left(x_{1}\right)=h\left(x_{0}\right)$, for all $\delta>0$ we have that $h\left(x_{1}\right)<h\left(x_{1}+\delta\right)$. Then considering 
$x_{1}$ in the place of $x_{0}$ above, the argument in (a) applies and it follows that there are more than one elements in the intersection of the balls under consideration, a contradiction again. This completes the proof of the assertion that if $B_{1 / 2}(f) \cap$ $B_{1 / 2}(g)$ is a singleton, then we necessarily have either

$$
f=\underline{0} \text { and } g=\underline{1} \text {, or } f=\underline{1} \text { and } g=\underline{0} \text {. }
$$

We proceed with the proof as follows. Since $\phi$ is an isometry we have that

$$
B_{1 / 2}(\phi(\underline{0})) \cap B_{1 / 2}(\phi(\underline{1}))=\phi\left(B_{1 / 2}(\underline{0}) \cap B_{1 / 2}(\underline{1})\right)
$$

which is a singleton. This implies that either $\phi(\underline{0})=\underline{0}$ and $\phi(\underline{1})=\underline{1}$, or $\phi(\underline{0})=\underline{1}$ and $\phi(\underline{1})=\underline{0}$. Let us assume the former case, i.e. that $\phi$ fixes $\underline{0}$ and $\underline{1}$.

For any $0 \leq a \leq b \leq 1$ denote by $\Delta_{a, b}$ the set

$$
\Delta_{a, b}=\left\{g \in \Delta(\mathbb{R}): \lim _{t \rightarrow-\infty} g(t)=a \text { and } \lim _{t \rightarrow \infty} g(t)=b\right\} .
$$

We assert that $\phi$ maps $\Delta_{a, b}$ onto itself. Indeed, it follows from the easy fact that $g \in \Delta(\mathbb{R})$ is an element of $\Delta_{a, b}$ if and only if $\rho(g, \underline{1})=1-a$ and $\rho(g, \underline{0})=$ $b$. Observe that we obtain in particular that $\phi$ leaves all constant functions invariant.

Now, let $a<b$ be two fixed but arbitrary elements of [0,1] and denote by $\phi_{a, b}$ the restriction of the transformation $\phi$ onto the subspace $\Delta_{a, b}$. It is apparent that the transformation

$$
f \mapsto \frac{\phi_{a, b}((b-a) f+a)-a}{b-a}, \quad f \in D(\mathbb{R})
$$

is a surjective isometry of $D(\mathbb{R})$. It follows from Theorem 1 that either there exists a strictly increasing bijection $\varphi_{a, b}: \mathbb{R} \rightarrow \mathbb{R}$ such that we have

$$
\frac{\phi_{a, b}((b-a) f(t)+a)-a}{b-a}=f\left(\varphi_{a, b}(t)\right), \quad t \in \mathbb{R}, f \in D(\mathbb{R})
$$

or there exists a strictly decreasing bijection $\psi_{a, b}: \mathbb{R} \rightarrow \mathbb{R}$ such that we have

$$
\frac{\phi_{a, b}((b-a) f(t)+a)-a}{b-a}=1-f\left(\psi_{a, b}(t)-\right), \quad t \in \mathbb{R}, f \in D(\mathbb{R}) .
$$

For an arbitrary element $F$ of $\Delta_{a, b}$ we see that $(F-a) /(b-a)$ is an element of $D(\mathbb{R})$. Insert this function into the corresponding equation (1) or (2). We get

$$
\phi_{a, b}(F)(t)=F\left(\varphi_{a, b}(t)\right), \quad t \in \mathbb{R}
$$

or

$$
\phi_{a, b}(F)(t)=a+b-F\left(\psi_{a, b}(t)-\right), \quad t \in \mathbb{R} .
$$

Recall that the numbers $a$ and $b$ above are fixed. Let us say that $\phi_{a, b}$ is of type I if it is of the form (3) with a strictly increasing bijection $\varphi_{a, b}: \mathbb{R} \rightarrow \mathbb{R}$ and let us say that $\phi_{a, b}$ is of type II if it is of the form (4) with a strictly decreasing bijection $\psi_{a, b}: \mathbb{R} \rightarrow \mathbb{R}$. 
Letting now $a$ and $b$ vary, we show that either all $\phi_{a, b}$ 's are of type I, or all of them are of type II. To verify this, first suppose that there exists $0<b<b^{\prime} \leq 1$ such that, for example, $\phi_{0, b}$ is of the form (3), i.e.

$$
\phi_{0, b}(f)(t)=f\left(\varphi_{0, b}(t)\right), \quad t \in \mathbb{R}, f \in \Delta_{0, b}
$$

and $\phi_{0, b^{\prime}}$ is of the form (4), i.e.

$$
\phi_{0, b^{\prime}}(f)(t)=b^{\prime}-f\left(\psi_{0, b^{\prime}}(t)-\right), \quad t \in \mathbb{R}, f \in \Delta_{0, b^{\prime}},
$$

where $\varphi_{0, b}: \mathbb{R} \rightarrow \mathbb{R}$ is a strictly increasing bijection and $\psi_{0, b^{\prime}}: \mathbb{R} \rightarrow \mathbb{R}$ is a strictly decreasing bijection. Let $p$ and $p^{\prime}$ be arbitrary real numbers. We consider Dirac-type generalized distribution functions $d_{p}^{b} \in \Delta_{0, b}$ and $d_{p^{\prime}}^{b^{\prime}} \in \Delta_{0, b^{\prime}}$ defined by

$$
d_{p}^{b}(t)=\left\{\begin{array}{ll}
0 & \text { if } t<p \\
b & \text { if } t \geq p
\end{array} \quad \text { and } \quad d_{p^{\prime}}^{b^{\prime}}(t)=\left\{\begin{aligned}
0 & \text { if } t<p^{\prime} \\
b^{\prime} & \text { if } t \geq p^{\prime}
\end{aligned}\right.\right.
$$

Suppose that $b^{\prime}-b<b$. We see that $p=p^{\prime}$ if and only if $\rho\left(d_{p}^{b}, d_{p^{\prime}}^{b^{\prime}}\right)=b^{\prime}-b$. Easy computation shows that

$$
\phi\left(d_{p}^{b}\right)=\phi_{0, b}\left(d_{p}^{b}\right)=d_{\varphi_{0, b}^{-1}(p)}^{b} \quad \text { and } \quad \phi\left(d_{p^{\prime}}^{b^{\prime}}\right)=\phi_{0, b^{\prime}}\left(d_{p^{\prime}}^{b^{\prime}}\right)=d_{\left.\psi_{0, b^{\prime}}^{-1} p^{\prime}\right)}^{b^{\prime}} .
$$

Since the distance between those functions must also be $b^{\prime}-b$, it follows that $\varphi_{0, b}^{-1}\left(p^{\prime}\right)=\psi_{0, b^{\prime}}^{-1}\left(p^{\prime}\right)$ for all $p^{\prime} \in \mathbb{R}$ which is an obvious contradiction. It is easy to see that we would arrive at a similar contradiction if we assumed that $\phi_{0, b}$ is of type II and $\phi_{0, b^{\prime}}$ is of type I. It follows that $\phi_{0, b}$ and $\phi_{0, b^{\prime}}$ are of the same type whenever $b^{\prime}-b<b$. Assume now that $b^{\prime}-b \geq b$. Then one can pick a finite sequence $b=b_{1}<b_{2}<\ldots<b_{n}=b^{\prime}$ such that $b_{i+1}-b_{i}<b_{i}$ holds for all $i=1, \ldots, n-1$. From what we have proved above, it follows step by step that the maps $\phi_{0, b_{1}}, \ldots, \phi_{0, b_{n}}$ are all of the same type. Therefore, we obtain that all maps $\phi_{0, b}, b \in[0,1]$ are of the same type. (Observe that since $\phi$ leaves the constant functions invariant, hence all $\phi_{a, a}, 0 \leq a \leq 1$ are both of type I and II.) In a similar fashion one can show that the maps $\phi_{a, 1}, 0 \leq a \leq 1$ are all of the same type, too. Still using the same idea, for any $0 \leq a \leq b<b^{\prime} \leq 1$ one can verify that $\phi_{a, b}$ and $\phi_{a, b^{\prime}}$ are of the same type and next that for any $0 \leq a<a^{\prime} \leq b$ the maps $\phi_{a, b}$ and $\phi_{a^{\prime}, b}$ are of the same type. From these one can easily conclude that all maps $\phi_{a, b}$ are of the same type, namely the type of $\phi_{0,1}$.

However, the above argument shows not only that the types of the maps $\phi_{a, b}$ are all the same but also that the inducing functions $\varphi_{a, b}$ or $\psi_{a, b}$ appearing in (3) or (4) are identical for all $a, b \in[0,1], a \leq b$. Indeed, first suppose that all $\phi_{a, b}$ are of type I. Let $0<b<b^{\prime} \leq 1$. Then $\phi_{0, b}$ is of the form

$$
\phi_{0, b}(f)(t)=f\left(\varphi_{0, b}(t)\right), \quad t \in \mathbb{R}, f \in \Delta_{0, b}
$$


and $\phi_{0, b^{\prime}}$ is of the form

$$
\phi_{0, b^{\prime}}(f)(t)=f\left(\varphi_{0, b^{\prime}}(t)\right), \quad t \in \mathbb{R}, f \in \Delta_{0, b^{\prime}},
$$

where the inducing functions $\varphi_{0, b}, \varphi_{0, b^{\prime}}: \mathbb{R} \rightarrow \mathbb{R}$ are strictly monotone increasing bijections. Assume that $b^{\prime}-b<b$. For the Dirac-type generalized distribution functions $d_{u}^{b}$ and $d_{u}^{b^{\prime}}$ we have

$$
\rho\left(d_{u}^{b}, d_{u}^{b^{\prime}}\right)=b^{\prime}-b, \quad u \in \mathbb{R}
$$

which implies that the distance between their images

$$
\phi\left(d_{u}^{b}\right)=d_{\varphi_{0, b}^{-1}(u)}^{b}, \quad \phi\left(d_{u}^{b^{\prime}}\right)=d_{\varphi_{0, b^{\prime}}^{-1}}^{b^{\prime}}(u)
$$

is also $b^{\prime}-b$ meaning that

$$
\rho\left(d_{\varphi_{0, b}^{-1}(u)}^{b}, d_{\varphi_{0, b^{\prime}}^{-1}(u)}^{b^{\prime}}\right)=b^{\prime}-b, \quad u \in \mathbb{R} .
$$

This gives us that $\varphi_{0, b}^{-1}(u)=\varphi_{0, b^{\prime}}^{-1}(u)$ holds for all $u \in \mathbb{R}$. This yields that the inducing functions $\varphi_{0, b}$ and $\varphi_{0, b^{\prime}}$ are identical. We can proceed exactly as with the types. Namely, if $b^{\prime}-b \geq b$, one can pick a finite sequence $b=b_{1}<b_{2}<$ $\ldots<b_{n}=b^{\prime}$ such that $b_{i+1}-b_{i}<b_{i}$ holds for all $i=1, \ldots, n-1$ and infer that the inducing functions of $\phi_{0, b_{1}}, \ldots, \phi_{0, b_{n}}$ are identical. As a consequence, we obtain that the inducing functions $\varphi_{0, b}$ are the same for all $b \in[0,1]$. In a similar fashion one can show that for every $a \in[0,1]$ the strictly monotone increasing bijections $\varphi_{a, 1}$ appearing in the form of $\phi_{a, 1}$ are identical. Still using the same idea, for any $0 \leq a \leq b<b^{\prime} \leq 1$ one can verify that $\varphi_{a, b}$ and $\varphi_{a, b^{\prime}}$ are the same and next that for any $0 \leq a<a^{\prime} \leq b$ the functions $\varphi_{a, b}$ and $\varphi_{a^{\prime}, b}$ are identical. From these one can easily conclude that the inducing functions of $\phi_{a, b}$ are all the same, namely, they equal the inducing function of $\phi_{0,1}$. Obviously, one can follow a similar argument in the case where all restricted maps $\phi_{a, b}$ are of type II.

Therefore, we have proved that either there exists a strictly increasing bijection $\varphi: \mathbb{R} \rightarrow \mathbb{R}$ such that

$$
\phi(f)(t)=f(\varphi(t)), \quad t \in \mathbb{R}, f \in \Delta(\mathbb{R})
$$

or there exists a strictly decreasing bijection $\psi: \mathbb{R} \rightarrow \mathbb{R}$ such that

$$
\phi(f)(t)=f_{-\infty}+f_{\infty}-f(\psi(t)-), \quad t \in \mathbb{R}, f \in \Delta(\mathbb{R}),
$$

where $f_{-\infty}=\lim _{t \rightarrow-\infty} f(t)$ and $f_{\infty}=\lim _{t \rightarrow \infty} f(t)$. However, this latter possibility can be ruled out easily. Indeed, consider for example the following generalized distribution functions:

$$
f(t)=\left\{\begin{array}{ll}
1 / 4 & \text { if } t<0 \\
1 / 2 & \text { if } t \geq 0
\end{array} \quad \text { and } \quad g(t)= \begin{cases}1 / 4 & \text { if } t<1 \\
3 / 4 & \text { if } t \geq 1 .\end{cases}\right.
$$

Then we have $\rho(f, g)=1 / 4$ while the distance between $\phi(f)$ and $\phi(g)$ would be $1 / 2$, a contradiction. Therefore, we have verified that in the case where $\phi$ leaves 
the constant functions $\underline{0}, \underline{1}$ invariant, the transformation $\phi$ is necessarily of the form $(5)$.

In the remains to treat the case where $\phi$ interchanges the constant functions $\underline{0}$ and $\underline{1}$. Then consider the transformation $\Psi: \Delta(\mathbb{R}) \rightarrow \Delta(\mathbb{R})$ defined by $\Psi(f)(t)=$ $1-\bar{f}((-t)-), t \in \mathbb{R}, f \in \Delta(\mathbb{R})$. It is easily seen to be a surjective isometry of $\Delta(\mathbb{R})$ which interchanges $\underline{0}$ and $\underline{1}$. Therefore, the transformation $\Psi \circ \phi$ is a surjective isometry which leaves $\underline{0}$ and $\underline{1}$ invariant. Applying the first part of the proof for this transformation, it follows that it is of the form (5). Then composing by $\Psi^{-1}=\Psi$ from the left, we easily get that $\phi$ is of the second form that appears in the statement of the theorem. The proof is complete.

In another recent paper [5] the first author has studied the KolmogorovSmirnov isometries of important subspaces of $D(\mathbb{R})$, too. The main result has been the description of the structure of all surjective isometries of the space $D_{c}(\mathbb{R})$ of all continuous distribution functions. It reads as follows.

Theorem 3. ([5]) Let $\phi: D_{c}(\mathbb{R}) \rightarrow D_{c}(\mathbb{R})$ be a surjective isometry with respect to the Kolmogorov-Smirnov metric, i.e. a bijective map with the property that

$$
\rho(\phi(f), \phi(g))=\rho(f, g)
$$

holds for all $f, g \in D_{c}(\mathbb{R})$. Then either there exists a strictly increasing bijection $\varphi: \mathbb{R} \rightarrow \mathbb{R}$ such that $\phi$ is of the form

$$
\phi(f)(t)=f(\varphi(t)), \quad t \in \mathbb{R}, f \in D_{c}(\mathbb{R})
$$

or there exists a strictly decreasing bijection $\psi: \mathbb{R} \rightarrow \mathbb{R}$ such that $\phi$ is of the form

$$
\phi(f)(t)=1-f(\psi(t)), \quad t \in \mathbb{R}, f \in D_{c}(\mathbb{R}) .
$$

Since the Dirac distribution functions are not present in the space $D_{c}(\mathbb{R})$, the author has used an approach which is completely different from the argument applied in the proof of Theorem 1 .

Let us denote by $\Delta_{c}(\mathbb{R})$ the set of all continuous generalized distribution functions. We conclude the present paper with the extension of Theorem 3 to the case of the surjective isometries of $\Delta_{c}(\mathbb{R})$ equipped with the KolmogorovSmirnov metric. The result is formally the same as for the surjective isometries of $D_{c}(\mathbb{R})$.

Theorem 4. Let $\phi: \Delta_{c}(\mathbb{R}) \rightarrow \Delta_{c}(\mathbb{R})$ be a surjective isometry with respect to the Kolmogorov-Smirnov metric. Then either there exists a strictly increasing bijection $\varphi: \mathbb{R} \rightarrow \mathbb{R}$ such that $\phi$ is of the form

$$
\phi(f)(t)=f(\varphi(t)), \quad t \in \mathbb{R}, f \in \Delta_{c}(\mathbb{R})
$$

or there exists a strictly decreasing bijection $\psi: \mathbb{R} \rightarrow \mathbb{R}$ such that $\phi$ is of the form

$$
\phi(f)(t)=1-f(\psi(t)), \quad t \in \mathbb{R}, f \in \Delta_{c}(\mathbb{R})
$$


Sketch of the proof. Our argument is rather similar to what we have applied in the proof of Theorem 2. Namely, we try to deduce the result from the corresponding statement Theorem 3 concerning the space $D_{c}(\mathbb{R})$ of all continuous distribution functions. We only sketch the argument.

First, one can verify that $B_{1 / 2}(f) \cap B_{1 / 2}(g)$ is a singleton if and only if $\{f, g\}=$ $\{\underline{0}, \underline{1}\}$. In fact, the proof of this characterization can go along the same lines as in the corresponding part of the proof of Theorem 2 with the difference that in the present case the situation is easier, several subcases do not appear due to the continuity of functions under consideration. Hence we obtain that $\phi$ either leaves the functions $\underline{0}$ and $\underline{1}$ invariant or interchanges them. Assume that we have the former case, i.e. $\phi$ fixes $\underline{0}$ and $\underline{1}$. We can continue as in the proof of Theorem 2 and show that for arbitrary $a, b \in[0,1], a \leq b$ the restriction $\phi_{a, b}$ of $\phi$ onto $\Delta_{c}(\mathbb{R}) \cap \Delta_{a, b}$ is a surjective isometry and then apply Theorem 3 to deduce that either there exists a strictly increasing bijection $\varphi_{a, b}: \mathbb{R} \rightarrow \mathbb{R}$ such that $\phi_{a, b}$ is of the form

$$
\phi_{a, b}(F)(t)=F\left(\varphi_{a, b}(t)\right), \quad t \in \mathbb{R}, F \in \Delta_{c}(\mathbb{R}) \cap \Delta_{a, b}
$$

or there exists a strictly decreasing bijection $\psi_{a, b}: \mathbb{R} \rightarrow \mathbb{R}$ such that $\phi_{a, b}$ is of the form

$$
\phi_{a, b}(F)(t)=b+a-F\left(\psi_{a, b}(t)\right), \quad t \in \mathbb{R}, F \in \Delta_{c}(\mathbb{R}) \cap \Delta_{a, b} .
$$

In the case (6) we say that $\phi_{a, b}$ is of type I while in the case (7) we say that it is of type II. In the proof of Theorem 2 we have employed Dirac-type generalized distribution functions to see that either all $\phi_{a, b}$ are type I or all of them are of type II, and moreover, that the inducing functions are all the same. At this point the present proof must be a bit changed due to the fact that the Dirac-type functions are not continuous. We argue as follows. Let $0<b<b^{\prime} \leq 1$. Suppose, for example, that $\phi_{0, b}$ is of type II and $\phi_{0, b^{\prime}}$ is of type I with inducing function $\psi_{0, b}$ and $\varphi_{0, b^{\prime}}$, respectively. Clearly, the former function is a strictly decreasing while the latter one is a strictly increasing bijection of $\mathbb{R}$. Pick two arbitrary real numbers $x<y$. Let $x<z<y$ be such that $b /(z-x)=b^{\prime} /(y-x)$. Consider the following continuous generalized distribution functions.

$$
f(t)=\left\{\begin{aligned}
0 & \text { if } t<x \\
\frac{b(t-x)}{z-x} & \text { if } x \leq t<z \\
b & \text { if } z \leq t
\end{aligned} \quad \text { and } \quad g(t)=\left\{\begin{aligned}
0 & \text { if } t<x \\
\frac{b^{\prime}(t-x)}{y-x} \quad & \text { if } x \leq t<y \\
b^{\prime} & \text { if } y \leq t
\end{aligned}\right.\right.
$$

Obviously, the distance between $f$ and $g$ is $b^{\prime}-b$. We assert that

$$
\psi_{0, b}^{-1}(y) \leq \varphi_{0, b^{\prime}}^{-1}(y)
$$

Indeed, in the opposite case there were a real number $t$ such that

$$
\psi_{0, b}^{-1}(y)>t>\varphi_{0, b^{\prime}}^{-1}(y)
$$


which would imply that $y<\psi_{0, b}(t)$ and $y<\varphi_{0, b^{\prime}}(t)$. By (6), (7) we would deduce that the distance between $\phi(f)$ and $\phi(g)$ is $b^{\prime}$, a clear contradiction. Therefore, we have $\psi_{0, b}^{-1}(y) \leq \varphi_{0, b^{\prime}}^{-1}(y)$ for all real numbers $y$ which is obviously untenable by the different monotonicity properties of $\psi_{0, b}$ and $\varphi_{0, b^{\prime}}$. One can apply a similar argument in the case where $\phi_{0, b}$ is of type I and $\phi_{0, b^{\prime}}$ is of type II. Then one can continue showing that the types of $\phi_{a, b}$ are all the same and next that the inducing functions are also identical. Just as in the proof of Theorem 2, we obtain that either there exists a strictly increasing bijection $\varphi: \mathbb{R} \rightarrow \mathbb{R}$ such that

$$
\phi(f)(t)=f(\varphi(t)), \quad t \in \mathbb{R}, f \in \Delta_{c}(\mathbb{R})
$$

or there exists a strictly decreasing bijection $\psi: \mathbb{R} \rightarrow \mathbb{R}$ such that

$$
\phi(f)(t)=f_{-\infty}+f_{\infty}-f(\psi(t)), \quad t \in \mathbb{R}, f \in \Delta_{c}(\mathbb{R}) .
$$

The second form for $\phi$ can be ruled out by choosing a pair of appropriate simple functions as in the proof of Theorem 2 modified to functions like $f$ and $g$ in (8) above. This completes the proof when $\phi$ fixes $\underline{0}$ and $\underline{1}$.

In the remaining case where $\phi$ interchanges the functions $\underline{0}$ and $\underline{1}$, we consider the transformation $\Psi$ on $\Delta_{c}(\mathbb{R})$ defined by $\Psi(f)(t)=1-f(-t), t \in \mathbb{R}, f \in \Delta_{c}(\mathbb{R})$ which is a surjective isometry interchanging $\underline{0}$ and $\underline{1}$. Composing $\phi$ by $\Psi$ from the left we obtain a surjective isometry on $\Delta_{c}(\mathbb{R})$ which leaves $\underline{0}$ and $\underline{1}$ invariant. Applying the first part of the proof for $\Psi \circ \phi$ we get that it is of the form (9). Composing by $\Psi^{-1}=\Psi$ from the left again, we obtain the second possible form for the original map $\phi$ that appears in the formulation of the theorem.

In the paper [5] results on the Kolmogorov-Smirnov isometries of the spaces of all absolute continuous or singular distribution functions have also been presented. However, since those concepts (absolute continuity and singularity) are not unambiguously defined for functions in $\Delta_{c}(\mathbb{R})$, here we do not discuss the corresponding extensions of the results in [5] for the setting of generalized distribution functions.

\section{REFERENCES}

[1] DOLINAR, G.-MOLNÁR, L.: Isometries of the space of distribution functions with respect to the Kolmogorov-Smirnov metric, J. Math. Anal. Appl. 348 (2008), 494-498.

[2] FELLER, W.: An Introduction to Probability Theory and its Applications, Vol. II, John Wiley and Sons, New York, 1971.

[3] FLEMINGR, R. J.-JAMISON, J. E.: Isometries on Banach spaces: function spaces. Vol. 1., Chapman \& Hall/CRC Monographs and Surveys in Pure and Applied Mathematics 129, Boca Raton, 2002.

[4] FLEMINGR, R. J.-JAMISON, J. E.: Isometries on Banach spaces: Vector-valued function spaces. Vol. 2., Chapman \& Hall/CRC Monographs and Surveys in Pure and Applied Mathematics 138, Boca Raton, 2007.

[5] MOLNÁR, L.: Kolmogorov-Smirnov isometries and affine automorphisms of spaces of distribution functions, Cent. Eur. J. Math. 9 (2011), 789-796. 
[6] SHIRYAEV, A. N.: Probability, Graduate Texts in Mathematics, No. 95, Springer-Verlag, 1996.

* Institute of Mathematics

University of DeBrecen

H-4010 Debrecen, P.O. Box 12

HUNGARY

E-mail address: molnarl@science.unideb.hu

URL: http://www.math. unideb.hu/ molnarl/

** Institute of Mathematics

University of Debrecen

H-4010 Debrecen, P.O. Box 12

HUNGARY

E-mail address: szokolp@science.unideb.hu 